\title{
Current-Loop Model for the Intermediate State of Type-I Superconductors
}

\author{
Raymond E. Goldstein ${ }^{1}$, David P. Jackson ${ }^{1 *}$, and Alan T. Dorsey ${ }^{2}$ \\ ${ }^{1}$ Department of Physics, Joseph Henry Laboratories, Princeton University, Princeton, NJ 08544 \\ ${ }^{2}$ Department of Physics, University of Virginia, McCormick Road, Charlottesville, VA 22901
}

(September 13, 2018)

\begin{abstract}
A theory is developed of the intricately fingered patterns of flux domains observed in the intermediate state of thin type-I superconductors. The patterns are shown to arise from the competition between the long-range BiotSavart interactions of the Meissner currents encircling each region and the superconducting-normal surface energy. The energy of a set of such domains is expressed as a nonlocal functional of the positions of their boundaries, and a simple gradient flow in configuration space yields branched flux domains qualitatively like those seen in experiment. Connections with pattern formation in amphiphilic monolayers and magnetic fluids are emphasized.
\end{abstract}

Typeset using REVTEX 
When a thin film of a type-I superconductor is placed in a magnetic field normal to the sample, the large demagnetizing effects associated with the film geometry preclude the establishment of the Meissner phase (with magnetic induction $\mathbf{B}=0$ ). The sample instead accommodates the field by breaking up into a large number of superconducting $(\mathbf{B}=0)$ and normal $(\mathbf{B} \neq 0)$ regions, usually forming very intricate patterns $[1]$. These arise in part from the competition between the magnetic field energy of the domains and the surface energy between the superconducting and normal regions. However, they have not been successfully explained theoretically, for virtually all theories of these patterns, starting from that of Landau [2], have explored this competition with variational calculations that assume regular geometries of the flux domains [3]. The hypothesized parallel stripes, ordered arrays of circles, etc. are rarely seen, the norm instead being the disordered patterns documented in classical experiments 田回, Moreover, the temperature and magnetic field history of the sample strongly influence the patterns, suggesting that they are likely not in a global energetic minimum.

Recent work in this area has emphasized that the diffusion of magnetic flux in the normal phase can influence the domain morphology [6]:7]. From asymptotic methods applied to the time-dependent Ginzburg-Landau (TDGL) model [8.9], a free-boundary representation for the motion of superconductor-normal (S-N) interfaces has been derived which is nearly identical to those describing the growth of a solid into a supercooled liquid, where the interface motion is unstable (producing dendrites, for example). By analogy it was suggested, and confirmed by numerical solution of the TDGL equations [6], that the growth of the superconducting phase into the supercooled normal phase should be dynamically unstable, leading to highly ramified domain shapes. While such diffusive instabilities may play a role in the pattern formation in the intermediate state, these studies are not directly applicable as they have all ignored demagnetizing effects.

Here we take a different approach to the intermediate state, ignoring entirely the diffusion of flux and focusing instead on the demagnetizing fields. We address the basic question: What is the energy of a thin multiply-connected superconducting domain, the normal regions 
of which are threaded with a magnetic field? A central issue is the degree to which the interactions between the Meissner currents, flowing along the S-N interfaces within the film, are screened by the superconducting regions. Pearl [10] and Fetter and Hohenberg [11] made the important observation that vortices in a thin film interact with a potential $V(r) \sim 1 / r$ for large separations $r$, while for small $r, V(r) \sim \ln (\Lambda / r)$, with $\Lambda$ an appropriate cut-off. Unlike in bulk, the interactions are unscreened, being dominated by the electromagnetic fields above and below the film. This suggests the simple model developed here: domains bounded by current loops interacting as in free space, endowed with line tension, and subject to the constraint of constant total magnetic flux through the sample. We focus on how such loops relax toward a local energy minimum when prepared in a nonequilibrium configuration. Such a model allows for the study of the shape of the domains, but does not readily account for changes in topology (e.g., fission). We conclude that the long-range interactions destabilize flux domains of regular shape, producing branched, fingered structures as seen in experiment.

Apart from the global flux constraint, this model is equivalent to one for domains of magnetic fluids [12] in Hele-Shaw flow [13, 14], where experiments [15, 16] show patterns like those observed in the intermediate state, with the same history-dependence noted earlier. Thin magnetic films [17] and monolayers of dipolar molecules [18] exhibit similar behavior, and are described by such models through the underlying similarity between electric and magnetic dipolar phenomena [19,20]. This model is also very similar to one introduced for pattern formation in certain reaction-diffusion systems [21]. There, fronts between regions of different chemical composition move in response to line tension and a nonlocal Biot-Savart coupling, and may exhibit labyrinthine patterns, as observed in recent experiments [22].

To begin, we note a crucial separation of length scales between the typical size of the flux domains (ca. $0.1 \mathrm{~mm}$ ) and the penetration depth $(\mathrm{ca} . \leq 1 \mu \mathrm{m})$ [1]. Thus on the scale of the patterns the superconductor-normal interface is sharp, and we may view the order parameter magnitude as piecewise constant. It follows that the energy is determined solely by the locations of the S-N interfaces.

In this macroscopic approach, the energy $\mathcal{F}$ of a configuration of flux domains arises 
from the bulk free energy of the two coexisting phases, the boundaries between them, and the magnetic field energy,

$$
\mathcal{F}=\mathcal{F}_{\text {bulk }}+\mathcal{F}_{\text {int }}+\mathcal{F}_{\text {field }}
$$

Suppose the film has thickness $d$, total area $A$, volume $V=A d$, and contains a set of normal domains $i$ with area $A_{i}$, length $L_{i}$, and whose boundary positions are $\mathbf{r}_{i}(s)$ (Fig. 11). We assume $\mathbf{r}_{i}$ is independent of $z$, neglecting the "fanning out" of the domains near the film surfaces [2]. The two phases occupy volumes $V_{s}$ and $V_{n}=d \sum_{i} A_{i}$, with $V_{s}+V_{n}=V$. Their bulk free energy densities $F_{s}$ and $F_{n}$ define the critical field $H_{c}(T)$ as $F_{n}-F_{s}=H_{c}^{2} / 8 \pi$. With $\rho_{n}=A_{n} / A$ the area fraction of the normal phase, $\sigma_{\mathrm{SN}}=\left(H_{c}^{2} / 8 \pi\right) \Delta$ the S-N interfacial tension (where $\Delta(T)$ can be interpreted as the interfacial width), we have

$$
\mathcal{F}_{\text {bulk }}=\left[F_{s}+\frac{H_{c}^{2}}{8 \pi} \rho_{n}\right] A d ; \quad \mathcal{F}_{\text {int }}=\frac{H_{c}^{2} d}{8 \pi} \Delta \sum_{i} L_{i} .
$$

The complexity of this problem lies entirely in the computation of the field energy. An applied field $\mathbf{H}=H_{a} \hat{\mathbf{e}}_{z}$, produces a field $H_{n} \hat{\mathbf{e}}_{z}$ in the normal regions, where $A H_{a}=A_{n} H_{n}$ by flux conservation, so

$$
H_{n}=\frac{H_{a}}{\rho_{n}}
$$

The requirement of tangential continuity of $\mathbf{H}$ across a $\mathrm{S}-\mathrm{N}$ interface gives the field in the superconducting region as $\mathbf{H}_{s}=H_{s} \hat{\mathbf{e}}_{z}=H_{n} \hat{\mathbf{e}}_{z}$. Taking the superconducting regions to be perfectly diamagnetic (with susceptibility $\chi=-1 / 4 \pi$ ) yields a magnetization $\mathbf{M}=$ $-\left(H_{n} / 4 \pi\right) \hat{\mathbf{e}}_{z}$, which guarantees that the magnetic induction in the superconducting regions vanishes: $\mathbf{B}_{s}=\mathbf{H}_{s}+4 \pi \mathbf{M}=0$. The field energy is that of domains of total magnetization $\mathbf{M}_{\text {tot }}$ (as yet unknown) in an external field $\mathbf{H}_{a}$,

$$
\mathcal{F}_{\text {field }}=-\frac{1}{2} \int d^{3} r \mathbf{M}_{\text {tot }} \cdot \mathbf{H}_{a}
$$

We quickly point out that if we ignore the fringing fields entirely and assume $\mathbf{M}_{\text {tot }}$ is completely uniform, the field energy would be 


$$
\mathcal{F}_{\text {field }}^{\prime}=-\frac{1}{2} V_{s}\left(-\frac{H_{n}}{4 \pi}\right) H_{a}=\frac{H_{a}^{2}}{8 \pi} \frac{1-\rho_{n}}{\rho_{n}} A d .
$$

Minimizing the sum $\mathcal{F}_{\text {bulk }}+\mathcal{F}_{\text {field }}^{\prime}$ sum with respect to $\rho_{n}$, we obtain the standard result [23] for the equilibrium value $\rho_{n}^{*}=H_{a} / H_{c}$; i.e., $H_{n}=H_{c}$. But we obtain no information about the shapes of the domains. The inclusion of line tension alters slightly the equilibrium area fraction, and renders the equilibrium domain shape a circle, as that has the minimum perimeter for a given area.

A field energy sensitive to the shape of the flux domains must account for fringing magnetic fields. Since the calculation of these is well-documented [13, 14], we summarize only the essential results. First write the total magnetization as $\mathbf{M}_{\text {tot }}=\chi \mathbf{H}_{\text {cap }}$, where $\mathbf{H}_{\text {cap }}$ is the magnetic field arising from the uniform magnetization $\chi \mathbf{H}_{n}$. The subscript "cap" indicates the equivalence of this problem to that of a uniformly charged parallel plate capacitor. Now write $\mathbf{H}_{\text {cap }}=\mathbf{H}^{(0)}+\mathbf{H}^{(1)}$, where $\mathbf{H}^{(0)}=H^{(0)} \hat{\mathbf{e}}_{z}$ is uniform between the plates and vanishes elsewhere. The choice $H^{(0)}=-4 \pi \chi H_{n}$ yields $\boldsymbol{\nabla} \cdot \mathbf{H}^{(1)}=0$, so $\mathbf{H}^{(1)}=\boldsymbol{\nabla} \times \mathbf{A}$, for some vector potential A. Choosing an appropriate gauge, we find $\nabla^{2} \mathbf{A}=\boldsymbol{\nabla} \times \mathbf{H}^{(0)}$, or

$$
\mathbf{A}(\mathbf{r})=-\frac{H_{n}}{4 \pi} \sum_{i} \int_{0}^{d} d z \oint d s \frac{\hat{\mathbf{t}}_{i}(s)}{\left|\mathbf{r}-\mathbf{r}_{i}(s, z)\right|},
$$

where $\hat{\mathbf{t}}_{i}(s)$ is the tangent to $\mathbf{r}_{i}(s)$. Substituting into (何), performing the integrations over the thickness of the slab [14], the energy of a set of interacting current loops is found to be

$$
\begin{aligned}
\mathcal{F}\left[\left\{\mathbf{r}_{i}\right\}\right]=\frac{H_{c}^{2} d}{8 \pi} & \left\{\Delta \sum_{i} L_{i}+A\left[\rho_{n}+\frac{h^{2}}{\rho_{n}}\right]\right. \\
& \left.-\frac{h^{2}}{4 \pi \rho_{n}} \sum_{i, j} \oint d s \oint d s^{\prime} \hat{\mathbf{t}}_{i} \cdot \hat{\mathbf{t}}_{j} \Phi_{i j}\right\},
\end{aligned}
$$

where $h=H_{a} / H_{c}$ is the reduced applied field, $\hat{\mathbf{t}}_{i}=\hat{\mathbf{t}}_{i}(s)$, and $\Phi_{i j}=\left(1+\delta_{i j}\right) \Phi\left(R_{i j} / d\right)$, with $R_{i j}=\left|\mathbf{r}_{i}(s)-\mathbf{r}_{j}\left(s^{\prime}\right)\right|$. The interaction potential

$$
\Phi(\xi)=\sinh ^{-1}(1 / \xi)+\xi-\sqrt{1+\xi^{2}},
$$

is Coulombic for $\xi \gg 1, \Phi \approx 1 /(2 \xi)$, reflecting the usual self-induction energy for elementary currents, while for $\xi \ll 1, \Phi \approx \ln \left(2 e^{-1} / \xi\right)$, with the film thickness $h$ acting as a cutoff (as in the vortex potential $V(r)$ above). 
The motion of S-N interfaces is determined by the normal component of the force acting at a point $\mathbf{r}_{i}(s)$ on a given interface. It is the negative functional derivative of the energy 9.14.

$$
\begin{aligned}
-\frac{1}{\sqrt{g_{i}}} \hat{\mathbf{n}}_{i} \cdot \frac{\delta \mathcal{F}}{\delta \mathbf{r}_{i}}= & \frac{H_{c}^{2} d}{8 \pi}\left\{-\Delta \mathcal{K}_{i}(s)+\Pi\right. \\
& \left.-\frac{h^{2}}{2 \pi \rho_{n} d} \sum_{j} \oint d s_{j}^{\prime} \hat{\mathbf{R}}_{i j} \times \hat{\mathbf{t}}_{j} \Phi_{i j}^{\prime}\right\},
\end{aligned}
$$

where $g_{i}=\partial_{\alpha} \mathbf{r}_{i} \cdot \partial_{\alpha} \mathbf{r}_{i}$ is the metric, $\mathcal{K}_{i}(s)$ the curvature, $\hat{\mathbf{n}}_{i}$ the unit normal, $\hat{\mathbf{R}}_{i j}=\mathbf{R}_{i j} /\left|\mathbf{R}_{i j}\right|$, $\Phi^{\prime}(\xi)=1-\left(1+\xi^{-2}\right)^{1 / 2}$, and $\Pi$ is an effective pressure given by

$$
\Pi=\frac{h^{2}}{\rho_{n}^{2}}\left[1-\frac{1}{4 \pi A} \sum_{i, j} \oint d s \oint d s^{\prime} \hat{\mathbf{t}}_{i} \cdot \hat{\mathbf{t}}_{j} \Phi_{i j}\right]-1 .
$$

Equation (9) reveals that the pattern formation arises from a competition between a magnetic pressure which incorporates flux conservation, the Young-Laplace force due to interfacial tension and the Biot-Savart force from the circulating currents. This additional long-range contribution to the boundary force has been noted before for magnetic fluids [13,14] and amphiphilic monolayers [24]. From extensive studies [13, 14, 15, 16, 20] of this competition in domains of fixed area, we know of the existence of branching instabilities of circular domains and buckling instabilities of stripes, phenomena which should carry over to the present problem with nonconserved area. Indeed, buckled domains are well known in type-I superconductors [4,5] and are seen as well in amphiphilic monolayers [25.

The simplest dynamical model for interface motion balances the normal component of the force against a viscous force localized on the interface, yielding an equation of motion 114

$$
\eta \hat{\mathbf{n}}_{i} \cdot \frac{\partial \mathbf{r}_{i}(s)}{\partial t}=-\frac{1}{\sqrt{g_{i}}} \hat{\mathbf{n}}_{i} \cdot \frac{\delta \mathcal{F}}{\delta \mathbf{r}_{i}}
$$

where $\eta$ is a kinetic coefficient. This equation of motion is local in time; in the present context it arises from the neglect of the diffusion of the magnetic flux in the normal phase [9], which is equivalent to assuming that the normal state conductivity is zero. The magnetic vector 
potential is then "slaved" to the order parameter, a limit considered also in the reactiondiffusion context [21]. To estimate $\eta$, we appeal to earlier results in the slaving limit for strongly type-I bulk superconductors [9] and obtain

$$
\eta=\frac{H_{c}^{2} d \Delta}{8 \pi} \frac{\pi \hbar}{8 k_{B} T_{c} \xi_{0}^{2}}
$$

where $T_{c}$ is the critical temperature and $\xi_{0}$ is the zero-temperature correlation length [26]. These results can be used to estimate the time scales for domain motion from other experimentally measured parameters.

The dynamical evolution of flux domain shapes governed by Eqs. (9)-(11) is a manybody problem of considerable computational complexity. To gain insight into its behavior, we consider here a mean-field description in which only the self-interaction of the current loops is considered; the Biot-Savart interactions between loops are neglected, while the flux-conservation condition is enforced by assigning the loop to a cell of area $A_{\text {cell }}$, with $\rho_{n}=A_{n} / A_{\text {cell }}$. By appropriate rescaling of the spatial variables, we deduce from Eqs. (9) and (10) that the location of the system in the $H-T$ plane is uniquely specified by the two dimensionless quantities $h$ and $\Delta / A_{\text {cell }}^{1 / 2}$.

To see the effects of the Biot-Savart interaction on the stability of a circular flux domain, Figures $2 \mathrm{a}$ and b show the evolution of a domain prepared with an area significantly less than the equilibrium value. In Fig. 2a the Biot-Savart coupling is omitted, and the circle simply relaxes to a new radius driven primarily by the magnetic pressure. Including the long-range interactions (Fig. 2b) results instead in the formation of a branched flux domain with threefold coordinated nodes, very similar to those seen in experiment [1, 1, 5]. In the early epoch the shape evolution is primarily a dilation with little change in shape, while the branching instabilities occur on a longer time scale. As found in previous studies [16], in the later stages of the shape evolution the driving force for the interfacial motion becomes extremely small, with very small energy differences between rather different shapes. This suggests that the interface motion would be extremely sensitive to external perturbations such as impurities or grain boundaries, which would then be effective in pinning the interfaces, not 
unlike the pinning of vortices in type-II superconductors [27. This may contribute to the history-dependence of the patterns discussed in the introduction.

Elsewhere we will discuss the derivation of the present model from the more fundamental TDGL equations by generalizations of existing asymptotic methods [8,9,21], as well as the details of stability analyses for various regular geometries. Given the connections outlined here between pattern formation in the intermediate state and in other systems such as amphiphilic monolayers and magnetic fluids, we suggest that it is of interest to extend experimental studies of flux domain shapes to probe systematically [17] the branching instabilities as a function of applied field and temperature.

We are grateful to T.C. Lubensky, N.P. Ong, and V.S. Pande for important comments at an early stage of this work, and thank A.O. Cebers, S. Erramilli, D.M. Petrich, M. Seul, M.J. Shelley, and T.A. Witten for numerous discussions. This work was supported in part by NSF Presidential Faculty Fellowship DMR 93-50227 (REG), NSF Grant DMR 92-23586 (ATD), and Alfred P. Sloan Foundation Fellowships (REG and ATD). 


\section{REFERENCES}

* Present address: Department of Physics, Dickinson College, Carlisle, PA 17013-2896.

[1] For an overview of experimental studies of the intermediate state, see R.P. Huebener, Magnetic Flux Structures in Superconductors (Springer-Verlag, New York, 1979).

[2] L.D. Landau, Sov. Phys. JETP 7, 371 (1937).

[3] E. R. Andrew, Proc. Roy. Soc. (London), A194, 98 (1948); K. Maki, Ann. Phys. 34, 363 (1965); G. Lasher, Phys. Rev. 154, 345 (1967); R. N. Goren and M. Tinkham, J. Low Temp. Phys. 5, 465 (1971); D. J. E. Callaway, Ann. Phys. 213, 166 (1992).

[4] F. Haenssler and L. Rinderer, Helv. Phys. Acta 40, 659 (1967).

[5] T.E. Faber, Proc. Roy. Soc. A 248, 460 (1958).

[6] H. Frahm, S. Ullah, and A.T. Dorsey, Phys. Rev. Lett. 66, 3067 (1991).

[7] F. Liu, M. Mondello, and N. Goldenfeld, Phys. Rev. Lett. 66, 3071 (1991).

[8] S. J. Chapman, Quart. Appl. Math. (to be published).

[9] A. T. Dorsey, Ann. Phys. 233, 248 (1994); J. C. Osborn and A. T. Dorsey, Phys. Rev. B (in press) (1994).

[10] J. Pearl, Appl. Phys. Lett. 5, 65 (1964).

[11] A.L. Fetter and P.C. Hohenberg, Phys. Rev. 159, 330 (1967).

[12] R.E. Rosensweig, Ferrohydrodynamics (Cambridge University Press, Cambridge, England, 1985).

[13] A.O. Tsebers and M.M. Mairov, Magnetohydrodynamics 16, 21 (1980).

[14] S.A. Langer, R.E. Goldstein, and D.P. Jackson, Phys. Rev. A 46, 4894 (1992); D.P. Jackson, R.E. Goldstein, and A.O. Cebers, Phys. Rev. E 50, 298 (1994). 
[15] R.E. Rosensweig, M. Zahn, and R. Shumovich, J. Magn. Magn. Mater. 39, 127 (1983).

[16] A.J. Dickstein, S. Erramilli, R.E. Goldstein, D.P. Jackson, and S.A. Langer, Science 261, $1012(1993)$.

[17] M. Seul, L.R. Monar, L.O'Gorman, and R. Wolfe, Science 254, 1616 (1991); M. Seul and D. Andelman, preprint (1994).

[18] K.Y.C. Lee and H.M. McConnell, J. Phys. Chem. 97, 9532 (1993).

[19] D. Andelman, F. Brochard, and J.-F. Joanny, J. Chem. Phys. 86, 3673 (1987).

[20] R.E. Goldstein and D.P. Jackson, J. Phys. Chem. 98, 9626 (1994).

[21] D.M. Petrich and R.E. Goldstein, Phys. Rev. Lett. 72, 1120 (1994); R.E. Goldstein, D.M. Petrich, and D.J. Muraki, preprint (1994).

[22] K.J. Lee, W.D. McCormick, Q. Ouyang, and H.L. Swinney, Science 261, 192 (1993).

[23] M. Tinkham, Introduction to Superconductivity (McGraw-Hill, New York, 1975), p. 95.

[24] D.A. Kessler and H. Levine, Phys. Rev. Lett. 67, 3121 (1991).

[25] K.J. Stine, C.M. Knobler, and R.C. Desai, Phys. Rev. Lett. 65, 1004 (1990).

[26] See A. A. Abrikosov, Fundamentals of the Theory of Metals (North-Holland, Amsterdam 1988), p. 479.

[27] P.W. Anderson and Y.B. Kim, Rev. Mod. Phys. 36, 39 (1964). 


\section{FIGURES}

FIG. 1. A thin slab of a type-I superconductor, of thickness $d$, viewed along the applied field $\mathbf{H}_{a}$. The normal regions are shown shaded. Adapted from 四.

FIG. 2. Numerical results from the gradient-flow model for the evolution of a flux domain boundary. Dashed initial condition is a circle of unit radius perturbed by low-order modes, evolving with $\Delta=0.01$ and $h=0.5$. (a) Rapid relaxation to a circle in the absence of long-range forces. (b) Rapid dilation followed by fingering on a much longer time scale in the presence of the Biot-Savart interactions. 